\title{
PERFIL E FUNCIONAMENTO DE COMITÊS MUNICIPAIS DE PREVENÇÃO DA MORTALIDADE MATERNA, INFANTIL E FETAL
}

\author{
PROFILE AND OPERATION OF CITY COMMITTEES FOR PREVENTION OF MATERNAL, INFANT AND FETAL \\ MORTALITY
}

\section{PERFILY Y FUNCIONAMIENTO DE COMITÉS MUNICIPALES PARA LA PREVENCIÓN DE LA MORTALIDAD MATERNA, INFANTIL Y FETAL}

Gabriela Souza França Lodi ${ }^{1}$, Ediene Dayane Lima², Liliane da Consolação Campos Ribeiro ${ }^{3}$, Helisamara Mota Guedes ${ }^{4}$

\section{RESUMO}

Objetivo: Analisar os comitês municipais de prevenção da mortalidade materna, infantil e fetal das regiões de saúde de Sete Lagoas e Curvelo- Minas Gerais quanto ao perfil dos seus membros e funcionamento. Método: Estudo de corte transversal em 35 comitês dos municípios jurisdicionados à Superintendência Regional de Saúde de Sete Lagoas. Foram aplicados questionários para as referências técnicas e secretários municipais de saúde, sendo a análise dos dados realizada por meio de estatística descritiva. Resultados: Encontrou-se formação predominante em enfermagem, média de idade acima de 30 anos e tempo no cargo superior a quatro anos. A maioria dos municípios possui comitê implantado, oficializado e atuante, mas muitos não realizam cronograma, nem registro das reuniões. Foram encontradas fragilidades no funcionamento, como inexistência de discussão entre os membros, não correção da causa básica do óbito, falta de divulgação dos dados e dos relatórios e ausência de atividades de mobilização social. Dentre os entraves, os mais citados foram: dificuldade de acesso a documentos para a investigação, falta de suporte da Superintendência Regional de Saúde e necessidade de capacitação. Conclusão: Existe a necessidade de qualificação das ações dos comitês, objetivando o aumento da sua efetividade na redução da mortalidade materna, infantil e fetal.

Descritores: Comitê de Profissionais, Mortalidade Materna, Mortalidade Infantil, Mortalidade Fetal.

\section{ABSTRACT}

Objective: to analyze the city committees for prevention of maternal, fetal and infant mortality in the health regions of Sete Lagoas and Curvelo - Minas Gerais, regarding the profile of their members and operation process. Method: cross-sectional study in 35 committees of the cities under the jurisdiction of the Regional Health Superintendence of Sete Lagoas. Questionnaires were applied to the technical references and municipal health secretaries. Data analysis was performed through descriptive statistics. Results: nursing education was predominant, mean age above 30 years and time in the post over four years. Most participants have an established, official and active committee. Nevertheless, many of them do not schedule or record meetings. There were weaknesses in the operation process, such as no discussion among members, no correction of cause of death, lack of disclosure of data and reports, absence of social mobilization activities. The most cited obstacles are difficult access to documents for investigation, lack of support from Regional Health Superintendence and the need for training. Conclusions: There is a need to qualify the actions of the committees, aiming to increase their effectiveness in reducing mortality.

Descriptors: Professional Staff Committees; Maternal Mortality; Infant Mortality; Fetal Mortality.

\section{RESUMEN}

Objetivo: analizar los comités municipales para la prevención de la mortalidad materna, infantil y fetal en las regiones de salud de Sete Lagoas y Curvelo - Minas Gerais con respecto al perfil de sus miembros y su funcionamiento. Método: estudio transversal en 35 comités de los municipios dentro de la jurisdicción de la Superintendencia Regional de Salud de Sete Lagoas. Se aplicaron cuestionarios a las referencias técnicas y secretarios de salud municipales, y el análisis de datos se realizó mediante estadística descriptiva. Resultados: hubo una capacitación predominante en enfermería, edad promedio de más de 30 años y tiempo en el cargo de más de cuatro años. La mayoría de los municipios tienen un comité establecido, oficial y activo, pero muchos no programan ni graban reuniones. Se encontró debilidad en el funcionamiento, ya que no hubo discusión entre los miembros, no se corrigió la causa básica de la muerte, falta de divulgación de datos e informes y ausencia de actividades de movilización social. Entre las barreras, las más citadas fueron la dificultad de acceso a documentos para investigación, la falta de apoyo de la Superintendencia Regional de Salud y la necesidad de capacitación. Conclusión: es necesario calificar las acciones de los comités, con el objetivo de aumentar su eficacia en la reducción de la mortalidad materna, infantil y fetal.

Descriptores: Comité de Profesionales; Mortalidad Materna; Mortalidad Infantil; Mortalidad Fetal.

${ }^{1}$ Enfermeira, Mestre em Ensino em Saúde. ${ }^{2}$ Acadêmica de Enfermagem da Universidade Federal do Vale do Jequitinhonha e Mucuri - UFVJM. Bolsista de Iniciação Científica da Fundação de Amparo à Pesquisa do Estado de Minas Gerais - FAPEMIG. ${ }^{3}$ Doutora em Ciências da Saúde. Docente do Departamento de Enfermagem e do Mestrado Profissional Ensino em Saúde UFVJM. ${ }^{4}$ Doutora em Enfermagem. Docente do Departamento de Enfermagem e Mestrado Ensino em Saúde UFVJM. 


\section{INTRODUÇÃO}

As primeiras experiências relacionadas à investigação do óbito e aos comitês de prevenção de morte materna ocorreram, no início do século $X X$, nos Estados Unidos da América ${ }^{(1-2)}$. No Brasil, a implantação dos comitês em nível hospitalar, municipal, regional e estadual consiste em uma estratégia do Ministério da Saúde, sendo adotada desde meados da década de $90^{(1-4)}$.

O primeiro manual com diretrizes para esse processo foi publicado, no ano de 2004, intitulado como Manual dos Comitês de Prevenção do Óbito Infantil e Fetal. Esse documento foi um marco e serviu como um orientador a todos os municípios brasileiros, na implantação dos seus comitês, sendo atualizado e republicado, no ano de $2009^{(1)}$.

A implantação e estruturação adequada dos comitês é uma estratégia para alcançar avanços na assistência à saúde materno-infantil, objetivando, ainda, a melhoria dos registros e estatísticas sobre a mortalidade, assim como a diminuição de mortes por causas evitáveis ${ }^{(1,5-6)}$. Assim, o seu efetivo funcionamento contribui para a redução da mortalidade materna, infantil e fetal, sendo esta uma meta de caráter internacional, que está incluída nos 17 Objetivos de Desenvolvimento Sustentável (ODS) e deve ser alcançada pelos países até $o$ ano de $2030^{(7)}$.

Os comitês devem ser interinstitucionais, multiprofissionais e possuir caráter técnicocientífico, educativo e formativo. Sua atuação deve ser sigilosa e é recomendado que tenham, em sua composição, representantes de instituições governamentais, da sociedade civil organizada e de universidades ligadas à saúde materno-infantil. As ações dos comitês não podem ser punitivas ou coercitivas, devendo ser exclusivamente educativas ${ }^{(1-2,4)}$.

A investigação dos óbitos maternos, fetais e infantis é a atividade fundamental dos comitês, sendo que o principal objetivo é identificar a evitabilidade dos casos e propor medidas preventivas, de acordo com as análises realizadas em cada um deles ${ }^{(8)}$. Dessa forma, a atuação dos comitês serve como um instrumento de gestão, capaz de dar visibilidade às questões da assistência materno-infantil e de gerar um pensamento crítico a respeito do tema ${ }^{(6,9)}$.

Relatório de auditoria do Tribunal de Contas da União (TCU) indicou que os comitês não se encontram em funcionamento em diversos Estados e na maioria dos municípios. Sendo que dos 24 comitês estaduais oficialmente implantados apenas 14 estavam atuantes. 0
Estado do Paraná foi apontado como a experiência mais positiva de atuação, existiam 22 comitês regionais e 160 comitês municipais, para um total de 399 municípios $^{(10)}$.

Existem diversos problemas em torno desse tema como o sub-registro que faz com que o coeficiente de morte por 100.000 mil nascidos vivos seja subestimado, pouca eficiência no processo de implementação e operacionalização dos comitês, sendo esta importante para a tomada de decisões na implantação e implementação de políticas públicas de saúde ${ }^{(11)}$.

As ações de vigilância ainda são pouco valorizadas como instrumento de planejamento das intervenções em saúde. É notório os baixos investimentos materiais e/ou pessoais, por parte dos gestores ${ }^{(12)}$ que preferem investir em ações assistencialistas. Dessa forma, perpetuam-se a morosidade na produção da informação e sua baixa qualidade, contribuindo para um processo que se reproduz de forma cíclica, indesejável e de difícil ruptura.

Em razão da relevância e ao impacto social do tema mortalidade materna, infantil e fetal, à necessidade constante de abordagem e de intervenções sobre esse problema e ao fato de existirem poucos dados sobre a atuação dos comitês, no Brasil( ${ }^{(2)}$ é que se propôs esse estudo. Assim, conduziu-se este trabalho, com o objetivo de analisar os comitês municipais de prevenção da mortalidade materna, infantil e fetal das regiões de saúde de Sete Lagoas e Curvelo- Minas Gerais quanto ao perfil dos seus membros e funcionamento.

\section{MÉTODO}

Trata-se de estudo de corte transversal, de abordagem quantitativa e descritiva, realizado, no período de outubro de 2017 a agosto de 2018, que utilizou, como objeto de análise, os 35 municípios sob a jurisdição da Superintendência Regional de Saúde (SRS) de Sete Lagoas, sendo 11 municípios da região de saúde de Curvelo e 24 municípios da região de saúde de Sete Lagoas. Os municípios foram delimitados, conforme o Plano Diretor de Regionalização (PDR) de Minas Gerais.

Nesses municípios, foram analisadas informações sobre o perfil e o funcionamento dos comitês de prevenção da mortalidade materna, infantil e fetal, por meio de questionários aplicados a dois grupos diferentes: secretários municipais de saúde e referências técnicas municipais dos comitês de cada um dos 
municípios. Na ausência de comitê municipal, o profissional da epidemiologia, responsável pela vigilância do óbito, foi o convidado a participar da pesquisa. Os questionários foram aplicados, via email e na SRS de Sete Lagoas, conforme a disponibilidade dos participantes.

Utilizaram-se de instrumentos de coleta validados por Dutra(12) que aborda questões relativas à implantação, atribuições e dificuldades dos comitês ou das equipes municipais responsáveis pelas suas atribuições e apoio da gestão municipal. A análise foi realizada, por meio do software Statistical Package for Social Sciences (SPSS), versão 25 . Foram realizadas análises de estatística descritiva para a obtenção de média, desvio padrão, frequências absolutas e relativas.

O estudo foi aprovado pelo Comitê de Ética de Pesquisa em Seres Humanos, da Universidade Federal dos Vales do Jequitinhonha e Mucuri, sob o número CAEE: 6865131760005108 autorizado pela Superintêndencia Regional de Saúde, e cumpriu os princípios éticos contidos na
Resolução no 466/2012 do Conselho Nacional de Saúde. Todos os respondentes do questionário assinaram o Termo de Consentimento Livre e Esclarecido (TCLE).

\section{RESULTADOS E DISCUSSÃO}

Da população total de 35 secretários e 35 referências técnicas, responderam ao questionário $34(97,1 \%)$ secretários municipais de saúde e $30(85,71 \%)$ referências técnicas dos comitês municipais de prevenção da mortalidade materna, infantil e fetal.

Em relação ao perfil dos secretários municipais $(n=34), 23(67,6 \%)$ eram do sexo feminino, 32(93,7\%) com idade superior a 30 anos, $18(52,9 \%)$ casados, $18(52,9 \%)$ estavam no cargo há mais de um ano. Sobre a escolaridade, 16 $(47,1 \%)$ possuíam especialização, 7 (20,6\%) ensino superior completo, $10(29,4 \%)$ formação em enfermagem (Tabela 1).

Tabela 1 - Distribuição da população de estudo de acordo com as variáveis demográficas, socioeconômicas e tempo de permanência no cargo, 2018( $n=64)$.

\begin{tabular}{|c|c|c|c|c|}
\hline \multirow[t]{2}{*}{ Variáveis } & \multicolumn{2}{|c|}{$\begin{array}{c}\text { Secretário } \\
(n=34)\end{array}$} & \multicolumn{2}{|c|}{$\begin{array}{l}\text { Referência técnica } \\
(n=30)\end{array}$} \\
\hline & $\mathbf{n}$ & $\%$ & $\mathbf{n}$ & $\%$ \\
\hline \multicolumn{5}{|l|}{ Sexo } \\
\hline Masculino & 11 & 32,4 & 1 & 3,3 \\
\hline Feminino & 23 & 67,6 & 29 & 96,7 \\
\hline \multicolumn{5}{|l|}{ Faixa etária (anos) } \\
\hline$\leq 29$ & 2 & 6,3 & 5 & 16,6 \\
\hline$>30$ & 32 & 93,7 & 25 & 83,4 \\
\hline \multicolumn{5}{|l|}{ Escolaridade } \\
\hline Ensino Médio & 4 & 11,8 & 2 & 6,6 \\
\hline Superior Incompleto & 4 & 11,8 & & \\
\hline Superior Completo & 7 & 20,6 & 15 & 50 \\
\hline Especialização & 16 & 47,0 & 12 & 40 \\
\hline Mestrado & 1 & 2,9 & 1 & 3,3 \\
\hline \multicolumn{5}{|l|}{ Formação } \\
\hline Enfermagem & 10 & 29,4 & 26 & 86,8 \\
\hline Outros profissionais da saúde & 8 & 23,6 & 2 & 6,6 \\
\hline Outros & 16 & 47 & 2 & 6,6 \\
\hline \multicolumn{5}{|l|}{ Tipo de Especialização } \\
\hline Area da Saúde & 10 & 29,4 & 13 & 56,5 \\
\hline Outros & 6 & 20,5 & 2 & 6,7 \\
\hline \multicolumn{5}{|l|}{ Tempo no cargo } \\
\hline$\leq 12$ meses & 3 & 8,8 & 7 & 23,4 \\
\hline$>12$ meses & 18 & 52,9 & 21 & 69,6 \\
\hline
\end{tabular}

Fonte: elaborada pelas autoras com base nos dados da pesquisa. Nota: as opcções menos citadas não foram inseridas na tabela.

Já, no grupo das referências técnicas $(n=30)$, $29(96,7 \%)$, eram do sexo feminino $25(83,2 \%)$ com idade superior a 30 anos, $17(56,7 \%)$ solteiros, $21(69,6 \%)$ estavam no cargo há mais de um ano.
Quanto à escolaridade $15(50,0 \%)$ possuiam ensino superior completo, 12 (40\%) possuíam especialização, sendo as referências técnicas, predominantemente, de enfermeiros $(n=26$; 


\section{6,8\%) (Tabela 1).}

O perfil dos profissionais é um dos fatores que influencia na implementação dos comitês. Sobre a faixa etária e o tempo no cargo dos secretários municipais de saúde e dos profissionais que exercem a função de referência técnica do comitê observa-se, neste estudo, que ambos os grupos possuem, em média, idade acima dos 30 anos e tempo no cargo superior a quatro anos. Esse achado também foi encontrado em outros estudos nacionais, que demonstraram que os comitês são formados, em grande parte, por profissionais com um maior tempo de experiência ${ }^{(2,15)}$.

Verificou-se que a formação em enfermagem, tanto no grupo dos secretários municipais de saúde quanto no das referências técnicas, foi a prevalente. Esse dado corrobora com o estudo feito, em Fortaleza, sobre os comitês hospitalares de prevenção de morte materna, no qual foi observado que eram constituídos, predominantemente, por enfermeiras ${ }^{(2)}$. A mesma realidade foi encontrada no município de Coronel Fabriciano, em Minas Gerais (MG) $)^{(15)}$. Tal fato pode estar relacionado aos projetos políticos pedagógicos dos cursos de graduação em enfermagem, que possibilitam uma formação profissional capacitada para atuação no Sistema Único de Saúde (SUS) ${ }^{(16)}$.

Todavia, é importante destacar que, conforme preconizado pelo Ministério da Saúde, os comitês devem ser multiprofissionais e interinstitucionais ${ }^{(1)}$. Dessa forma, a participação de outras categorias profissionais e de diversas instituições enriquece as discussões sobre os óbitos, na medida em que diferentes situações de risco podem ser identificadas pelos variados pontos de vista ${ }^{(2)}$.

Na Tabela 2, aborda-se a existência dos comitês. Houve divergências nas informações, conforme se pode observar. Para os secretários de saúde participantes, 32 (94,1\%) municípios possuem comitê, enquanto que, para as referências técnicas, em 26 (86,7\%) municípios, eles estão implantados. Sobre a atuação dos comitês municipais de forma efetiva, 28 (93,3\%) referências técnicas consideram que os comitês cumprem com o seu papel, enquanto para 25 $(73,5 \%)$ secretários eles são totalmente atuantes. Os enfermeiros da Estratégia Saúde da Família (ESF) foram citados também como responsáveis pelas investigações dos óbitos, por $7(20,6 \%)$ secretários e $14(46,7 \%)$ referências técnicas.

Tabela 2 - Existência dos Comitês Municipais de Prevenção da Mortalidade Materna, Infantil e Fetal- Regiões de Saúde de Sete Lagoas e Curvelo, 2018.

\begin{tabular}{|c|c|c|c|c|}
\hline \multirow[t]{2}{*}{ Itens de implantação do comitê } & \multicolumn{2}{|c|}{$\begin{array}{c}\text { Secretário } \\
(n=34)\end{array}$} & \multicolumn{2}{|c|}{$\begin{array}{l}\text { Referência técnica } \\
(n=30)\end{array}$} \\
\hline & $\mathbf{n}$ & $\%$ & $\mathbf{n}$ & $\%$ \\
\hline \multicolumn{5}{|l|}{ Existência do comitê no município } \\
\hline Não & & & 2 & 6,7 \\
\hline Sim & 32 & 94,1 & 26 & 86,7 \\
\hline Não sei & & & 2 & 6,7 \\
\hline Em andamento & 2 & 5,9 & 0 & 0,0 \\
\hline \multicolumn{5}{|l|}{ Atuação efetiva do comitê municipal } \\
\hline Sim & 25 & 73,5 & 28 & 93,3 \\
\hline Em parte & 9 & 26,5 & 2 & 6,7 \\
\hline \multicolumn{5}{|c|}{$\begin{array}{l}\text { Outros profissionais responsáveis pelas investigações dos óbitos } \\
\text { maternos, infantis e fetais } * \text {; a }\end{array}$} \\
\hline Enfermagem ESF & 7 & 20,6 & 14 & 46,7 \\
\hline Vigilância Epidemiológica & 3 & 8,9 & 7 & 23,3 \\
\hline
\end{tabular}

Fonte: elaborada pelas autoras com base nos dados da pesquisa.

* mais de uma resposta por indivíduo. aalternativas menos citadas não foram inseridas na tabela.

Apenas $6,7 \%$ das referências, relataram a ausência do comitê em seu município, dado expressivamente menor que o percentual de comitês não implantados encontrado em um estudo na Região Ampliada de Saúde Jequitinhonha de $M G$, que foi de $42,4 \%^{(15,17)}$. Essa mesma pesquisa, realizada no ano de 2015, constatou que $26,4 \%$ dos existentes eram atuantes, número inferior ao encontrado nesse trabalho, que retrata uma atuação efetiva da maior parte deles. Outro estudo, também realizado, no ano de 2015, nos municípios 
jurisdicionados à SRS de Belo Horizonte/MG, apresentou um índice de $63,2 \%$ de comitês existentes na região ${ }^{(7)}$.

Pode-se verificar, na Tabela 3 , que a investigação da causa base do óbito e a redução da taxa de mortalidade materna, infantil e fetal foram as opções mais citadas como missão primordial dos comitês, segundo 28 (82,4\%) secretários de saúde. Na percepção de 14 (46,7\%) referências técnicas, a principal missão dos comitês também é diminuir a taxa de mortalidade materna, infantil e fetal.
Quanto aos entraves para o funcionamento do comitê, a maior parte dos profissionais dos dois grupos relatou que eles não existem. Entretanto, alguns participantes citaram dificuldades enfrentadas, sendo que $8(23,5 \%)$ secretários consideram a ausência de comitê e a dificuldade de acesso aos dados hospitalares como as principais. Já no grupo das referências, os entraves mais citados foram equipes desmotivadas ou não empenhadas, omissão de dados hospitalares e falta de suporte da SRS, sendo cada um deles referenciado por $7(23,3 \%)$ profissionais (Tabela $3)$.

Tabela 3 - Atribuições e Dificuldades encontradas pelos Comitês Municipais de Prevenção da Mortalidade Materna, Infantil e Fetal- Regiões de Saúde de Sete Lagoas e Curvelo, 2018.

\begin{tabular}{|c|c|c|c|c|}
\hline \multirow[t]{2}{*}{ Variáveis } & \multicolumn{2}{|c|}{$\begin{array}{c}\text { Secretário } \\
(n=34)\end{array}$} & \multicolumn{2}{|c|}{ Referência técnica $(\mathrm{n}=30)$} \\
\hline & $\mathbf{n}$ & $\%$ & $\mathbf{n}$ & $\%$ \\
\hline \multicolumn{5}{|l|}{ Missão primordial do comitê*;a } \\
\hline Diminuir a taxa de mortalidade materna, fetal e infantil & 28 & 82,4 & 14 & 46,7 \\
\hline Investigar causa base do óbito & 28 & 82,4 & 3 & 10,0 \\
\hline Realizar critério de evitabilidade & 21 & 61,8 & 2 & 6,6 \\
\hline Levantar/propor soluções & 23 & 67,6 & 13 & 43,3 \\
\hline \multicolumn{5}{|l|}{ Entraves na investigação dos óbitos ${ }^{a}$} \\
\hline Não & 25 & 73,5 & 20 & 66,7 \\
\hline Sim & 8 & 23,5 & 7 & 23,3 \\
\hline Não sabe & 1 & 2,9 & 2 & 6,7 \\
\hline \multicolumn{5}{|l|}{ Se sim, quais? ${ }^{*} ; \mathrm{a}$} \\
\hline Ausência de comitê & 8 & 23,5 & 2 & 6,7 \\
\hline Dificuldades de acesso aos dados hospitalares & 8 & 23,5 & 3 & 10,0 \\
\hline Óbitos fora do município de residência & 6 & 17,6 & 4 & 13,4 \\
\hline Equipes desmotivadas ou não empenhadas & 1 & 2,9 & 7 & 23,3 \\
\hline Omissão de dados hospitalares & 2 & 5,8 & 7 & 23,3 \\
\hline Falta de suporte da SRS & 0 & 0,0 & 7 & 23,3 \\
\hline
\end{tabular}

Fonte: elaborada pelas autoras com base nos dados da pesquisa.

* mais de uma resposta por indivíduo. aalternativas menos citadas não foram inseridas na tabela.

No que tange ao apoio que é dado pela gestão ao funcionamento dos comitês municipais e à realização da investigação dos óbitos, 28 $(82,4 \%)$ secretários consideram esse apoio presente, enquanto apenas $14(46,7 \%)$ referências técnicas acreditam existir esse auxílio. Ainda assim, $28(82,4 \%)$ dos secretários e $21(70 \%)$ das referências técnicas relatam haver necessidade de um maior favorecimento da gestão aos comitês. A capacitação dos profisionais atuantes é a alternativa mais citada pelos dois grupos (Tabela 4).

Tabela 4 - Apoio da Gestão aos Comitês Municipais de Prevenção da Mortalidade Materna, Infantil e FetalRegiões de Saúde de Sete Lagoas e Curvelo, 2018.

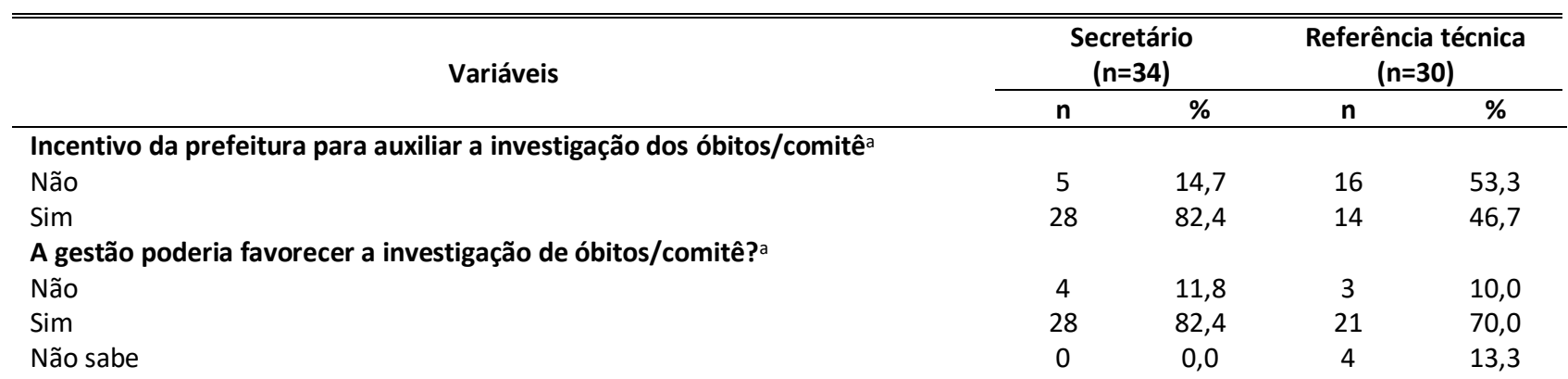




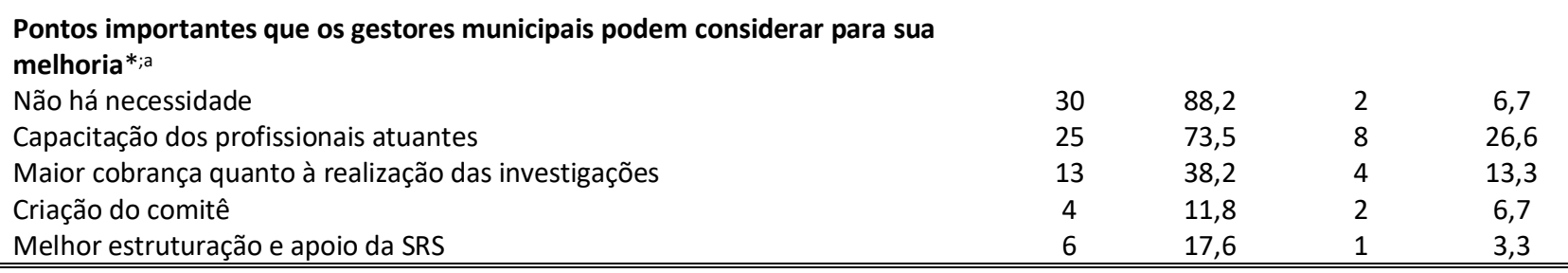

*mais de uma resposta por indivíduo. aalternativas menos citadas não foram inseridas na tabela.

Fonte: elaborada pelas autoras com base nos dados da pesquisa.

A necessidade de capacitação também é citada em outros estudos $^{(7,17-18)}$. Pesquisa feita na Zona da Mata de Minas Gerais apresentou como problema recorrente à capacitação profissional. Também foram citadas como entraves, naquela região, a falta de reconhecimento da atividade de vigilância do óbito pelos gestores; a insuficiência de tempo para a realização da investigação dos óbitos; a precariedade de recursos e de infraestrutura e a ausência de comprometimento dos membros ${ }^{(19)}$.

Algumas perguntas de cunho técnico sobre os comitês de prevenção da mortalidade foram realizadas apenas para as referências técnicas, visando a conhecer, de forma mais aprofundada, o seu funcionamento. Assim, quando questionadas sobre o que entendem como "investigação do óbito", 28 (93,3\%) das referências responderam que está relacionada à redução da taxa de mortalidade. Levantar soluções foi uma alternativa citada por 21 (70\%) das referências e identificar o critério de evitabilidade dos óbitos foi uma opção citada por $20(66,7 \%)$ desses profissionais.

Embora tenha aparecido com menor frequência, o entendimento de que a investigação do óbito seria uma atividade apenas para cumprimento de metas, ainda existe, sendo essa uma alternativa citada por $8 \quad(26,6 \%)$ dos profissionais. Esse achado corrobora com o estudo realizado na região do Vale Jequitinhonha (MG), no qual o cumprimento de metas foi citado como atribuição do comitê pela maior parte dos municípios $^{(20)}$. Tal fato pode estar relacionado à existência de legislações que preconizam a realização da investigação dos óbitos pelos serviços de saúde e também à existência de indicadores, atrelados ao recebimento de recursos financeiros, que estabelecem porcentagens mínimas obrigatórias de investigação oportuna dos óbitos maternos, infantis e fetais e de mulheres em idade fértil para os municípios ${ }^{(20-22)}$.

É importante salientar que Portarias do Ministério da Saúde (MS) determinam que a vigilância do óbito materno, infantil e fetal é uma atividade obrigatória nos serviços de saúde que integram o SUS, sejam eles públicos ou privados. Esses óbitos são eventos de investigação obrigatória, cujo objetivo é identificar os seus fatores causais e auxiliar na proposição de medidas que contribuam para evitar a ocorrência de novos $\operatorname{casos}^{(20,23)}$. Dessa forma, pela investigação dos óbitos é possível analisar a assistência à saúde prestada. Essas constatações possibilitam a qualificação dos serviços, na medida em que são definidas as práticas que devem ou não ser alteradas ${ }^{(24)}$.

Sobre a operacionalização dos comitês, 28 $(93,3 \%)$ das referências técnicas, quando questionadas se o comitê municipal está oficializado, responderam que sim, ou seja, a maioria. O tipo de documento prevalente para a formalização do comitê foi o ofício de composição dos membros, citado por 24 (80\%) dos profissionais (Tabela 5).

Tabela 5 - Operacionalização dos Comitês Municipais de Prevenção da Mortalidade Materna, Infantil e FetalRegiões de Saúde de Sete Lagoas e Curvelo, 2018.

\begin{tabular}{|c|c|c|}
\hline Variáveis & $\mathrm{n}=\mathbf{3 0}$ & $\%$ \\
\hline \multicolumn{3}{|l|}{ Oficialização do Comitê } \\
\hline Sim & 28 & 93,3 \\
\hline Parcialmente/em andamento & 2 & 6,7 \\
\hline \multicolumn{3}{|l|}{ Documentos de formalização utilizados* } \\
\hline Portaria & 7 & 23,3 \\
\hline Regimento & 18 & 60,0 \\
\hline
\end{tabular}




\begin{tabular}{lcc} 
Ofício de composição dos membros & 24 & 80,0 \\
Resolução & 3 & 10,0 \\
Existência de cronograma de reuniões & & 46,7 \\
Não & 14 & 53,3 \\
Sim & 16 & 23,3 \\
Se sim, qual a periodicidade das reuniões? & 7 & 3,3 \\
De acordo com a demanda & 1 & 10,0 \\
Mensal & 3 & 16,6 \\
Bimestral & 5 & 23,3 \\
Trimestral & & 76,7 \\
Existência de registro oficial (ata) das reuniões & 7 & 13,3 \\
Não & 23 & 13,3 \\
Sim & & 6,7 \\
Se não, que outro tipo de documento é utilizado?* & 4 & 2 \\
Não há registros & 4 & \\
Livro de Registros & 2 & \\
Outros & & \\
\hline
\end{tabular}

Fonte: elaborada pelas autoras com base nos dados da pesquisa.

*mais de uma resposta por indivíduo.

Ainda, na Tabela 5, pode-se observar que 16 $(53,3 \%)$ dos profissionais relatam possuir cronograma para a realização de reuniões. Entretanto, dos municípios que possuem calendário, 7 (23,3\%) dizem realizar as reuniões de acordo com a demanda. Com relação à forma de registro das reuniões, 23 (76,7\%) relatam existir registro oficial em ata. Dentre os que não possuem ata, $4(13,3 \%)$ relatam não haver nenhum tipo de registro para documentar a reunião.

O MS determina que os comitês devam ser oficializados, por meio de portaria ou resolução publicadas pela secretaria de saúde e também por um regimento interno ${ }^{(1)}$. Nas regiões estudadas, verifica-se que a maior parte dos comitês é oficializada, situação diferente da encontrada em outra região do Estado de Minas Gerais, onde apenas metade dos municípios possui comitê oficializado ${ }^{(7)}$. Porém, apesar de oficializados, muitos comitês não possuem cronograma de reuniões nem realizam registro das mesmas em ata. O MS e a Secretaria de Saúde do Estado definem que os comitês devem elaborar o seu regimento interno, contendo todas as suas normas de funcionamento e suas rotinas de trabalho, incluindo a forma de registro das reuniões ${ }^{(1,25)}$. Todavia, em alguns dos comitês analisados, não é feito nenhum tipo de registro. Essa prática deve ser modificada para que a realização do trabalho possa ser efetivamente comprovada e também para que as atividades desenvolvidas não se percam com o passar do tempo e com a rotatividade profissional.

Em grande parte dos municípios, não há discussão conjunta entre os membros do comitê para aplicar os critérios de evitabilidade, ficando essa função concentrada em apenas um profissional. É importante pontuar que todos os quatro municípios que relataram não realizar ou realizar, parcialmente, os critérios de evitabilidade atribuíram esse fato à falta de capacitação sobre o tema (Tabela 6).

Tabela 6 - Processos de Trabalho dos Comitês Municipais de Prevenção da Mortalidade Materna, Infantil e fetal- Regiões de Saúde de Sete Lagoas e Curvelo, 2018.

\begin{tabular}{lcc}
\hline \hline \multicolumn{1}{c}{ Variáveis } & $\mathbf{n}=\mathbf{3 0}$ & $\%$ \\
\hline É realizado pelo comitê o critério de evitabilidade para os óbitos? & 2 & 6,7 \\
Não & 26 \\
Sim & 2 & 6,7 \\
Em parte & 6,7 \\
Se não ou em parte, por quê? & 4 \\
Não houve treinamento & 26 \\
Não se aplica & 86,7 \\
Há discussão com todos os membros e em conjunto para realizar o critério de evitabilidade?a & 12 \\
Não & 40,0 \\
Sim & 17 \\
Se não, por quê? & 56,7
\end{tabular}


Apenas um membro realiza

Falta de tempo

Equipe não entrosada

Nem todos sabem realizar o critério

Se procurada a Superintendência Regional de Saúde, conseguem sanar as dúvidas quanto à

investigação de óbito/comitês?

Sim

Às vezes

Dificuldade de acesso aos documentos para realizar a investigação de óbito

Não

Sim

Após a investigação do óbito é feita a correção da causa básica no SIM, se necessário?

Não

Sim

Não Sabe

De que forma é feita a divulgação dos dados obtidos pelo comitê?*;a

Encaminhado para SRS

Não é divulgado

Reuniões

Encaminhado para SMS

Boletins

Relatórios

Fóruns

Envio de relatório contendo a análise final dos casos para Comitê Regional/Estadual

Não

Sim

Realização de mobilização da sociedade sobre a prevenção dos óbitos maternos, fetais e infantis?

Não

Sim

Se sim, qual periodicidade?

De acordo com a demanda

Outros

Mensal

Fonte: elaborada pelas autoras com base nos dados da pesquisa.

* mais de uma resposta por indivíduo. aalternativas menos citadas não foram inseridas na tabela.

Com relação às dúvidas existentes a respeito da investigação do óbito e do funcionamento dos comitês, 29 (96,7\%) dos profissionais responderam que conseguem sanálas ao procurar a Superintendência Regional de Saúde (Tabela 6). É importante salientar que a regional de saúde exerce importante papel de articuladora e apoiadora dos municípios, no que diz respeito à implantação das políticas de saúde e contribuição para uma gestão de saúde pública coparticipativa $^{(17)}$. Assim, a SRS deve estar sempre atenta às necessidades dos municípios sob sua jurisdição, buscando sempre ofertar capacitações, monitorar as atividades desenvolvidas e os indicadores de saúde em prol de uma melhoria na prestação dos serviços.

A existência de dificuldade de acesso aos documentos necessários para realizar a investigação do óbito está presente em 19 (63,3\%) dos municípios. Esse parece ser um problema frequente em outras regiões do Brasil, como no Vale Jequitinhonha-MG, que possui estudo, citando as dificuldades de acesso aos prontuários médicos e às declarações de óbito (DO) preenchidas, adequadamente, e, no Ceará, cuja pesquisa referiu problemas em acessar prontuários hospitalares, fichas de encaminhamento e laudos de necropsia, por parte dos comitês daquela região $0^{(17,18)}$.

Ainda relacionado ao processo de trabalho dos comitês e considerando o seu papel de auxiliar na qualificação das informações, é importante destacar que, embora a maioria deles realize a correção da causa básica do óbito no Sistema de Informação de Mortalidade (SIM), após a investigação $(83,3 \%)$ dos municípios, alguns dos entrevistados não realizam ou não sabem dizer se essa atividade é executada. Estudo das regiões de saúde jurisdicionadas à SRS Belo Horizonte mostrou que muitos dos municípios não realizam essa correção ${ }^{(7)}$. Pesquisa do Vale Jequitinhonha, MG, relacionou a dificuldade da maioria dos municípios inserirem ou corrigirem dados no SIM após conclusão da investigação à falta de 
treinamento $^{(17)}$.

Outra constatação que reflete a fragilidade no processo de trabalho dos comitês pesquisados é a falta de divulgação dos dados obtidos por eles. A respeito dessa divulgação, 17 (45,9\%) dos municípios dizem encaminhar relatórios para a SRS. Entretanto, 6 (16,2\%) não realizam nenhum tipo de divulgação e 19 (63,3\%) comitês municipais não enviam relatórios periódicos que contenham o consolidado dos casos investigados e as medidas de prevenção formuladas para o comitê regional/estadual. Além disso, 19 (63,3\%) dos municípios também não realizam nenhum tipo de mobilização da sociedade a respeito da prevenção dos óbitos maternos, fetais e infantis. Dentre os $11(36,7 \%)$ comitês que fazem esse tipo de ação, 7 (63,6\%) realizam essas atividades, de acordo com a demanda, não existindo uma periodicidade pré-determinada.

Estudo realizado em Recife, sobre a vigilância do óbito infantil detectou que esse processo é constituído de quatro fases, sendo elas: identificação e triagem dos casos elegíveis; investigação epidemiológica, por meio de fichas próprias, contendo todas as variáveis recomendadas pelo Ministério da Saúde; discussão dos óbitos, que verifica qual o nível de interferência do setor saúde, na ocorrência daqueles casos e, por último, elaboração e encaminhamento de propostas de intervenção, por meio de relatórios, para as áreas responsáveis e correção dos sistemas de informação(25).

Esse processo vai de encontro às sugestões do MS, que recomenda que a investigação do óbito deva ser realizada pelo setor de vigilância epidemiológica com o objetivo de identificar os seus fatores determinantes. Em seguida, um resumo realizado sobre cada caso, pelos profissionais da vigilância, deve ser apresentado nas reuniões dos comitês para discussão aprofundada entre os diversos profissionais e setores participantes. Por meio dessa análise é que são levantadas as falhas ocorridas, aplicados os critérios de evitabilidade, realizadas as propostas para evitar a reincidência de casos evitáveis, além de verificar a necessidade de melhoria nos registros e de correções nos sistemas de informação ${ }^{(1,25)}$.

Portanto, a elaboração de um documento com recomendações preventivas após análise dos óbitos e o seu encaminhamento para os gestores de saúde de todos os níveis se constitui como a etapa final do processo de trabalho dos comitês. Para garantir efetividade, essas ações devem ser institucionalizadas e continuadas e, como forma de garantir a mobilização social, os resultados devem ser divulgados para as diversas entidades científicas, governamentais, não governamentais e para a sociedade civil ${ }^{(15)}$.

Uma limitação desse estudo refere-se ao fato da coleta de dados ter sido realizada por meio de aplicação de questionário. A participação, in loco, nas reuniões dos comitês municipais e a análise de documentos oferecidos por eles poderia contribuir para evitar viés de informação por parte dos respondentes.

\section{CONCLUSÃO}

Os achados do estudo permitiram identificar diversas deficiências e dificuldades nos processos de trabalho dos comitês, o que indica a existência de inadequações em seu funcionamento. Esse fato alerta sobre a necessidade de qualificação das ações dos comitês, objetivando o aumento da sua efetividade na redução da mortalidade materna, infantil e fetal.

Os comitês, quando adequadamente estruturados, permitem a identificação dos fatores que levaram ao óbito e a proposição de medidas de intervenção para melhorar a qualidade da assistência materno-infantil, colaborando, dessa forma, com a construção e avaliação das políticas públicas de saúde vigentes.

Acredita-se que a pesquisa servirá para embasar as Secretarias Municipais de Saúde (SMS) e a SRS, na tomada decisão para implantação de ações que visem à melhoria do processo de trabalho dos comitês e da realização das investigações de óbitos maternos, infantis e fetais nos municípios.

Sugere-se que novos estudos sobre a temática sejam realizados. Nesse sentido, seria interessante analisar outras regiões do estado de Minas Gerais e do Brasil no que diz respeito à implantação, funcionamento e, principalmente, sobre a efetividade desses comitês.

\section{REFERÊNCIAS}

1- Brasil, Ministério da Saúde. Manual de vigilância do óbito infantil e fetal e do comitê de prevenção do óbito infantil e fetal. Brasília (DF): Ministério da Saúde; 2009.

2- Cruz HP, Pessoa SMF, Machado MMT, Feitosa HN, Carvalho FHC. Comitês hospitalares de prevenção de morte materna em Fortaleza: Perfil e funcionamento. Rev Bras Promoç Saúde 
2015;28(2):223-9.

DOI:

10.5020/18061230.2015.p223

3- Dias BAS, Santos Neto ET, Andrade MAC. Classificações de evitabilidade dos óbitos infantis: Diferentes métodos, diferentes repercussões? Cad Saúde Pública 2017;33 (5):1-15. DOI: 10.1590/0102-311x00125916

4- Vega CEP, Soares VNM, Nasr AMLF. Mortalidade materna tardia: Comparação de dois comitês de mortalidade materna no Brasil. Cad Saúde Pública 2017; 33 (3):1-13. DOI: 10.1590/0102-311x00197315

5- Santos HG, Andrade SM, Silva AMR, Carvalho WO, Mesas AE, González AD. Concordância sobre causas básicas de morte infantil entre registros originais e após investigação: Análise de dois biênios nos anos 2000. Rev Bras Epidemiol 2014;17(2):313-22. DOI: 10.1590/18094503201400020003ENG

6- Oliveira CM, Guimarães MJB, Bonfim CV, Frias PG, Antonino VCS, Guimarães ALS, et al. Adequação da investigação dos óbitos infantis no Recife, Pernambuco, Brasil. Ciênc Saúde Coletiva 2018;23(3):701-14. DOI: 10.1590/141381232018233.14052016

7- Rodrigues LA. Avaliação do processo de investigação e análise de óbitos maternos, fetais e infantis em uma região de saúde de Minas Gerais, Brasil [dissertação]. Belo Horizonte: Escola de Enfermagem da Universidade Federal de Minas Gerais; 2016.

8- Minas Gerais, Secretaria de Estado de Saúde. Manual de orientações para comitês de prevenção do óbito fetal e infantil. Belo Horizonte: SES; 2004. 9- Santos SPC, Lansky S, Ishitani LH, França EB. Óbitos infantis evitáveis em Belo Horizonte: Análise de concordância da causa básica, 20102011. Rev Bras Saúde Mater Infant. 2015;15(4):389-99. DOI: 10.1590/S151938292015000400003

10- Tribunal de Contas da União. Relatório de auditoria de natureza operacional. Brasília (DF): TCU; 2017.

11- Instituto Brasileiro de Geografia e Estatística. Brasil em síntese. Brasília (DF): IBGE; $2020 . \quad$ Available in: http://brasilemsintese.ibge.gov.br/populacao/tax as-de-mortalidade-infantil.html

12- Melo CM, Aquino TIS, Soares MQ, Bevilacqua PD. Death surveillance as an indicator of the quality of health care for women and children. Ciênc Saúde Coletiva 2017;22(10):3457-65. DOI: 10.1590/1413-812320172210.19652017
13- Dutra IR, Andrade GN, Rezende EM, Gazzinelli, A. Investigação dos óbitos infantil e fetal no Vale do Jequitinhonha, Minas Gerais, Brasil. Rev Min Enferm. 2015;19(3):605-11. DOI: 10.5935/1415-2762.20150046

14- Dutra I R. Vigilância do óbito fetal e infantil na Região Ampliada de Saúde Jequitinhonha, Minas Gerais [tese]. Belo Horizonte: Escola de Enfermagem da Universidade Federal de Minas Gerais; 2015.

15- Santos CA, Silva T, Lima EC. Trajetória de um comitê de mortalidade materna. Rev Enfermagem Integrada 2009; 2(2):333-44.

16- Winters JRF, Prado ML, Heidemann ITSB. A formação em enfermagem orientada aos princípios do Sistema Único de Saúde: Percepção dos formandos. Esc Anna Nery 2016;20(2):248-53. DOI: 10.5935/1414-8145.20160033

17- Dutra IR, Andrade GN, Rezende EM, Gazzinelli A. Investigação dos óbitos infantil e fetal no Vale do Jequitinhonha, Minas Gerais, Brasil. Rev Min Enferm. 2015;19(3): 597-604. DOI: 10.5935/14152762.20150046

18- Araújo, LB Análise da implantação e operacionalização dos comitês de prevenção do óbito infantil e fetal no Estado do Ceará [dissertação] Fortaleza: Universidade Federal do Ceará; 2012.

19- Melo CM, Aquino TIS, Soares MQ, Bevilacqua PD. Vigilância do óbito como indicador da qualidade da atenção à saúde da mulher e da criança. Ciênc Saúde Coletiva 2017;22(10):345765. DOI: 10.1590/1413-812320172210.19652017 20- Brasil, Ministério da Saúde. Portaria no 1119, de 05 de junho de 2008. Regulamenta a vigilância de óbitos maternos. Diário Oficial da União 2008.

21- Careti CM, Scarpelini A, Furtado M. Perfil da mortalidade infantil a partir da investigação de óbitos. Rev Eletr Enf. 2014;16(2):352-60. DOI: 10.5216/ree.v16i2.20321

22- Minas Gerais, Secretaria de Estado da Saúde. Resolução SES/MG № 5.484, de 17 de novembro de 2016. Estabelece normas gerais para participação, execução, acompanhamento, monitoramento e avaliação do Programa de Monitoramento das Ações de Vigilância em Saúde, no âmbito do Estado de Minas Gerais. Diário Oficial de Minas Gerais 2016.

23- Brasil, Ministério da Saúde. Portaria n. 72, de 11 de janeiro de 2010. Estabelece que a vigilância do óbito infantil e fetal é obrigatória nos serviços de saúde (públicos e privados) que integram o Sistema Único de Saúde (SUS). Brasília. Diário Oficial de Minas Gerais 2010. 
24- Minas Gerais, Secretaria de Estado de Saúde. Resolução no 5016, de novembro de 2015. Restabelece a organização dos Comitês Estadual, Regionais, Municipais, Compartilhados e Hospitalares de Prevenção de Mortalidade Materna, Infantil e Fetal e dá outras providências. Diário do Executivo 2015.

25- Oliveira, C M, Bonfim CV, Guimarães MJB, Frias PG, Antonino VCS, Medeiros ZM. Vigilância do óbito infantil no Recife, Pernambuco: operacionalização, potencialidades e limites. Epidemiol Serv Saúde 2017;26(2):413-9. DOI: 10.5123/s1679-49742017000200019

Nota: Extraído da Dissertação de Mestrado Profissional Ensino em Saúde da Universidade Federal dos Vales do Jequitinhonha e Mucuri: Análise dos comitês municipais de prevenção da mortalidade materna, infantil e fetal nas Regiões de Saúde de Sete Lagoas e Curvelo-MG.

Recebido em: 21/10/2019

Aprovado em: 07/05/2020

\section{Endereço de Correspondência:}

Liliane da Consolação Campos Ribeiro

Rua da Glória,187. Centro. Diamantina, Minas Gerais.

e-mail: lilianeribeiro@hotmail.com 